\title{
Cáncer de pulmón en el área sanitaria de Pontevedra: incidencia, presentación clínica y supervivencia
}

\section{Lung cancer in the Pontevedra health area: incidence, clinical presentation and survival}

\author{
F.J. González-Barcala ${ }^{1}$, J.A. Falagan ${ }^{2}$, J.M. García-Prim ${ }^{3}$, L. Valdes ${ }^{1}$, J.M. Carreira ${ }^{4}$, \\ A. Puga ${ }^{5}$, M.T. García-Sanz ${ }^{6}$, D. Antón ${ }^{7}$, J.C. Canive ${ }^{7}$, A. Pose ${ }^{8}$, R. López-López ${ }^{9}$
}

\section{RESUMEN}

Fundamento. Describir las características clínicas, epidemiológicas y supervivencia de los pacientes con cáncer de pulmón (CP) en el área sanitaria de Pontevedra.

Material y métodos. Se realizó un estudio observacional, retrospectivo, incluyendo todos los pacientes con diagnóstico de CP en el área sanitaria del Complexo Hospitalario de Pontevedra (CHOP), incluyendo todos los casos diagnosticados en un período de 3 años. De cada paciente se incluyeron edad, sexo, tabaquismo, comorbilidad, estado funcional, método diagnóstico, tipo histológico, estadio, tipo de tratamiento y supervivencia. Los pacientes fueron seguidos durante 3 años

Resultados. Se incluyeron en el estudio un total de 358 casos de $\mathrm{CP}$, lo cual supone una tasa cruda de incidencia ajustada a la población europea estándar de 37,33/100.000 habitantes/año en varones y de 4,88/100.000 habitantes/año en mujeres. El 87\% fueron varones, de 68,7 años de edad media, el $82 \%$ fumadores o exfumadores. El tipo histológico más frecuente fue el epidermoide, con el $35,2 \%$ de los casos. En el $79 \%$ de los casos se realizó el diagnóstico en estadios III-B o IV. La quimioterapia fue el primer tratamiento en el $53 \%$ de los casos. La supervivencia al primer año es del $25 \%$, que se reduce al $4 \%$ al tercer año.

Conclusiones. La incidencia de CP en nuestra área sanitaria sigue predominando en varones fumadores, aunque la incidencia en el sexo femenino y en nunca fumadores es superior a otras poblaciones de nuestro entorno. El diagnóstico se lleva a cabo en fases avanzadas de la enfermedad y la supervivencia es pobre.

Palabras clave. Cáncer de pulmón. Diagnóstico. Pronóstico. Incidencia. Tratamiento.

\begin{abstract}
Backgroud. To describe the clinical and epidemiologica characteristics, and survival of patients with lung cancer (LC) in the Pontevedra Health Area.

Methods. A retrospective observational study was conducted on all patients with a diagnosis of LC in the Pontevedra Hospital Complex (CHOP) health area over a three-year period. The data recorded included, age, gender, smoking history, comorbidity, functional status, diagnostic method, histology type, stage, treatment received, and survival. The patients were followed up for 3 years.
\end{abstract}

Results. A total of 358 cases of LC were included in the study, which gave a crude incidence rate adjusted to the standard European population of 37.33/100,000 inhabitants/ year in males and 4.88/100,000 inhabitants/year in females. The large majority were males (87\%). The mean age was 68.7 years, and $82 \%$ were smokers or ex-smokers. The most common histology type was epidermoid, with $35.2 \%$ of the cases. The diagnosis was made in stages III-B or IV in $79 \%$ of cases. Chemotherapy was the first treatment in $53 \%$ of the cases. Survival after the first year was $25 \%$, which decreased to $4 \%$ at the third year.

Conclusions. The incidence of LC in our health area continues to be mainly in male smokers, although the incidence in females and in people who never smoked is higher than in other populations in our country. It is diagnosed in the advance stages of the disease, and survival is poor.

Key words. Lung cancer. Diagnosis. Prognosis. Incidence. Treatment.
1. Servicio de Neumología. Hospital Clínico-Universitario. Santiago de Compostela.

2. Servicio de Documentación Clínica. Hospital de Pontevedra.

3. Servicio de Cirugía Torácica. Hospital Clínico-Universitario. Santiago de Compostela.

4. Departamento de Radiología. Universidad de Santiago de Compostela.

5. Departamento de Enfermería. Universidad de Santiago de Compostela.

6. Servicio de Urgencias. Hospital do Salnes

7. Medicina de Familia. Hospital de Pontevedra.

8. Servicio de Medicina Interna. Hospital Clínico-Universitario. Santiago de Compostela.

9. Servicio de Oncología. Hospital Clínico-Universitario. Santiago de Compostela.

\section{Correspondencia:}

F.J. González-Barcala

Servicio de Neumología

Hospital Clínico Universitario

C/ Choupana s/n

15706 Santiago de Compostela

E-mail: francisco.javier.gonzalez.barcala@sergas.es

Recepción: 11 de enero de 2013

Aceptación provisional: 20 de febrero de 2013

Aceptación definitiva: 18 de marzo de 2013 


\section{INTRODUCCIÓN}

El cáncer de pulmón (CP) es un problema de salud relevante por su elevada incidencia y mortalidad. En España continúa siendo la primera causa de mortalidad por cáncer en varones, y la tercera en mujeres, tras el cáncer de mama y el colorrectal ${ }^{1}$. La previsión para los próximos años parece favorable en los hombres, con tendencia a reducirse la incidencia de la enfermedad, pero en el sexo femenino tiende a aumentar significativamente ${ }^{2}$.

Para evaluar la importancia de cada enfermedad en una población determinada es esencial el conocimiento de algunos indicadores básicos, que nos permiten definir prioridades en el manejo de la enfermedad, identificar áreas de investigación, y establecer medidas de control ${ }^{2}$. Esto es especialmente importante en patologías como el $\mathrm{CP}$, donde se observan importantes variaciones en el pronóstico entre poblaciones cercanas. En un estudio español reciente se refiere que el $\mathrm{CP}$ es el tumor que presenta mayor variabilidad en la supervivencia entre las diferentes zonas geográficas, siendo la supervivencia a 5 años el doble en Navarra que en Granada ${ }^{3}$.

La distribución de lo distintos tipos histológicos presenta diferencias entre zonas geográficas y sexos. En España el tipo histológico más frecuente es el epidermoi$\mathrm{de}^{4-10}$; el adenocarcinoma muestra una clara tendencia a incrementar su frecuencia relativa en Europa ${ }^{11}$, y se ha convertido en el más frecuente en la población de USA ${ }^{12}$.

El objetivo de este trabajo es describir las características clínicas, citohistológicas y epidemiológicas del CP en el área sanitaria del hospital de Pontevedra, así como la supervivencia entre los años 2005 y 2008.

\section{MATERIAL Y MÉTODOS}

Se ha realizado un estudio observacional, retrospectivo, incluyendo todos los pacientes con diagnóstico de $\mathrm{CP}$ en el área sanitaria del Complexo Hospitalario de Pontevedra (CHOP), que abarca un área con una población de 307.916 habitantes.

A partir de los datos aportados por el servicio de Documentación Clínica del
CHOP, se han identificado los pacientes con CP diagnosticados en el período de estudio, entre el 1 de junio de 2005 y el 31 de mayo de 2008. Fueron incluidos en el estudio aquellos casos con confirmación citohistológica de CP. Asimismo, también se incluyeron pacientes sin diagnóstico citohistológico, pero con características clínico-radiológicas compatibles en los cuales se excluyeron razonablemente otros diagnósticos ${ }^{4}$.

De cada paciente se incluyeron edad, sexo, tabaquismo, comorbilidad, estado funcional, método diagnóstico, tipo histológico, estadio, tipo de tratamiento y supervivencia. Los pacientes fueron seguidos durante 3 años.

La clasificación histológica se realizó según las pautas de la Organización Mundial de la Salud de $1982^{13}$. La estadificación tumoral TNM se realizó en base a la propuesta por Mountain ${ }^{14}$, aceptada por la Sociedad Española de Neumología y Cirugía Torácica ${ }^{15}$.

En función del tabaquismo se establecieron 3 categorías mutuamente excluyentes, nunca fumador, fumador activo, y exfumador, según lo referido en la historia clínica por los médicos responsables de la asistencia del paciente.

La comorbilidad se determinó por medio de la escala de comorbilidad de Charlson ${ }^{16} ;$ y el estadio funcional mediante la escala del Eastern Cooperative Oncology Group (ECOG) ${ }^{17}$.

La fecha de fallecimiento se obtuvo de la historia clínica del paciente o por los datos del registro de mortalidad de Galicia.

La base de datos se elaboró sin datos personales identificatiavos de los pacientes excepto la edad y el sexo, para garantizar el manejo anonimizado.

\section{ANÁLISIS ESTADÍSTICO}

Las variables categóricas se expresaron mediante frecuencias y valores absolutos. Las variables continuas se mostraron utilizando la media \pm desviación típica cuando siguen distribución normal, y con la mediana y el rango intercuartílico cuando la distribución resulta ser no gaussiana. La norma- 
lidad de la distribución se verificó mediante la prueba de Kolmogorov-Smirnov.

El análisis de supervivencia se realizó mediante la prueba de Kaplan-Meier. Para comparar la supervivencia entre diferentes grupos se utilizó la prueba de log rank y la de Breslow como alternativa de ésta cuando se evidenciaba una divergencia del azar proporcional.

Se consideró significativo un valor de $\mathrm{p}<0,05$. El análisis se realizó con el paquete estadístico SPSS 15.0. El estudio fue aprobado por el Comité Ético de Galicia.

\section{RESULTADOS}

Fueron incluidos en el estudio un total de 358 casos de $\mathrm{CP}$, lo cual supone una tasa cruda de incidencia de 70/100.000 habitantes/año en varones y de 9,43/100.000 habitantes/año en mujeres. Las tasas ajustadas a la población europea mundial fueron de $37,33 / 100.000$ habitantes/año en varones y de 4,88/100.000 habitantes/año en el sexo femenino como se muestra en la tabla 1 donde también se refleja la tasa ajustada a la población europea.

Tabla 1. Incidencia (casos/100.000 habitantes /año)

\begin{tabular}{lccc}
\hline & Varones & Mujeres & Total \\
\hline Tasa cruda & 70,00 & 9,43 & 38,75 \\
\hline Incidencia ajustada a la Población Mundial Estándar & 37,33 & 4,88 & 19,33 \\
\hline Incidencia ajustada a la Población Europea & 55,39 & 6,81 & 28 \\
\hline
\end{tabular}

El $87,4 \%$ de nuestros pacientes eran varones, con una edad media de 68,7 años (rango de 32 a 98 años; desviación estándar de 11,9 años); el 82\% eran fumadores o exfumadores y el 75\% tenían alguna comorbilidad evaluada por el índice de Charlson (Tabla 2).

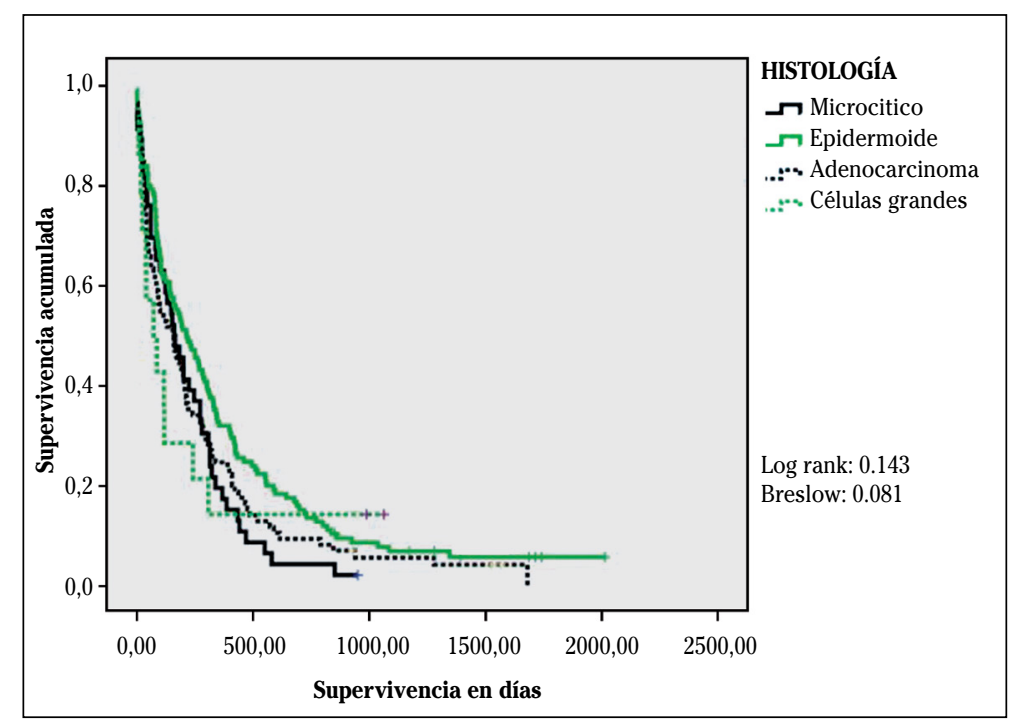

Figura 1. Supervivencia en función de la histología. 
Tabla 2. Características demográficas y de comorbilidad de los pacientes incluidos

\begin{tabular}{|c|c|c|}
\hline & & n (\%) \\
\hline Sexo & $\begin{array}{l}\text { Varón } \\
\text { Mujer }\end{array}$ & $\begin{array}{r}313(87,4) \\
45(12,6)\end{array}$ \\
\hline Edad & $\begin{array}{l}<50 \text { años } \\
50-59 \text { años } \\
60-69 \text { años } \\
70-79 \text { años } \\
\text { >=80 años }\end{array}$ & $\begin{array}{r}21(5,9) \\
59(16,5) \\
96(26,8) \\
121(33,8) \\
61(17,0)\end{array}$ \\
\hline Tabaquismo & $\begin{array}{l}\text { Nunca fumador } \\
\text { Ex fumador } \\
\text { Fumador activo }\end{array}$ & $\begin{array}{r}63(17,6) \\
155(43,3) \\
140(39,1)\end{array}$ \\
\hline Índice de Charlson & $\begin{array}{r}0 \\
1 \\
2 \\
\geq 3\end{array}$ & $\begin{array}{r}89(24,9) \\
100(27,9) \\
72(20,1) \\
97(27,1)\end{array}$ \\
\hline ECOG & $\begin{array}{l}0 \\
1 \\
2 \\
3 \\
4\end{array}$ & $\begin{array}{r}37(10,3) \\
197(55,0) \\
88(24,6) \\
24(6,7) \\
12(3,4)\end{array}$ \\
\hline Total de casos & & 358 \\
\hline
\end{tabular}

n: número de pacientes

ECOG: Escala Eastern Cooperative Oncology Group

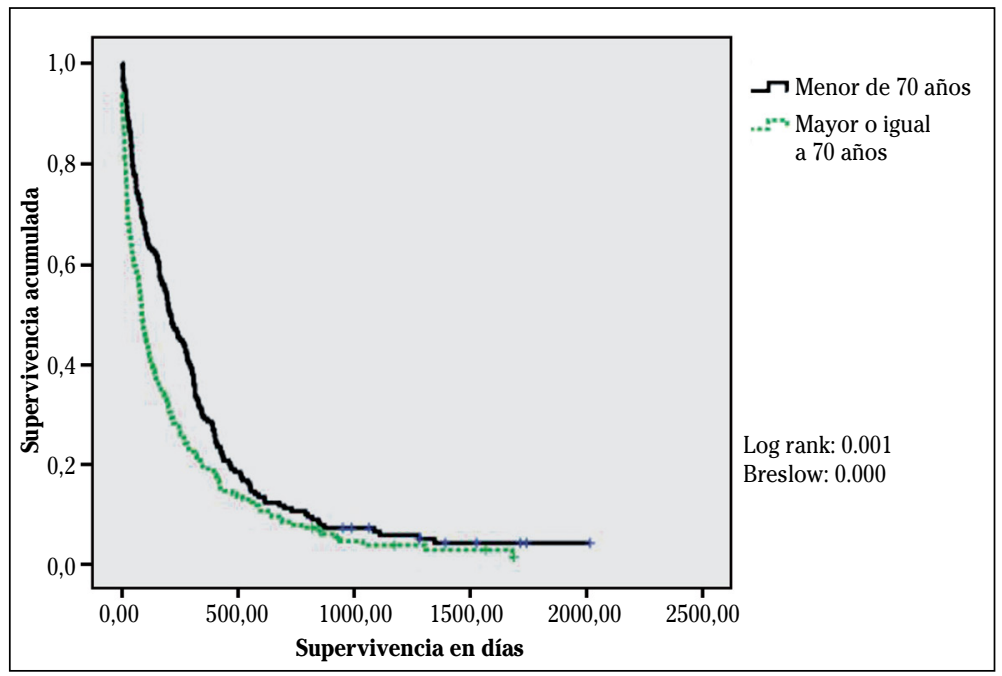

Figura 2. Supervivencia en función de la edad (menor de 70 años versus mayores de 70 años). 
Los diagnósticos se realizaron en fases avanzadas de la enfermedad, estando el $79 \%$ de pacientes en estadio III-B o IV. El método de diagnóstico más frecuente fue la fibrobroncoscopia (FBC), en el 41,6\% de los casos. El tipo histológico más frecuente fue el epidermoide, con el $35,2 \%$ de los pacientes. En el 12,8\% de casos el diagnóstico fue clínico-radiológico, sin conseguir la confirmación cito-histológica. Como primer tratamiento, el más utilizado fue la quimioterapia, aplicada al 53\% de pacientes. Se realizó cirugía como primera opción terapéutica en el $3,7 \%$ de casos (ninguno en pacientes con CP células pequeñas), y en el $32,4 \%$ se instauró tratamiento paliativo como opción terapéutica inicial (Tabla 3).

Tabla 3. Descripción del método diagnóstico, presentación clínica, tratamiento y supervivencia

\begin{tabular}{|c|c|}
\hline & n (\%) \\
\hline \multicolumn{2}{|l|}{ Método diagnóstico } \\
\hline Clínico-radiológico & $46(12,8)$ \\
\hline Fibrobroncoscopia & $149(41,6)$ \\
\hline PAAF transtorácica & $86(24)$ \\
\hline Adenopatía periférica & $19(5,3)$ \\
\hline Técnica pleural & $23(6,4)$ \\
\hline Cirugía torácica & $4(1,1)$ \\
\hline Otras & $30(8,4)$ \\
\hline \multicolumn{2}{|l|}{ Tipo histológico } \\
\hline Clínico-radiológico & $46(12,8)$ \\
\hline Microcítico & $46(12,8)$ \\
\hline Epidermoide & $126(35,2)$ \\
\hline Adenocarcinoma & $85(23,7)$ \\
\hline Células grandes & $14(3,9)$ \\
\hline Otros & $51(11,6)$ \\
\hline \multicolumn{2}{|l|}{ Estadio } \\
\hline I-A & $7(2,0)$ \\
\hline $\mathrm{I}-\mathrm{B}$ & $25(7,0)$ \\
\hline II-A & $5(1,4)$ \\
\hline II-B & $4(1,1)$ \\
\hline III-A & $34(9,6)$ \\
\hline III-B & $105(29,5)$ \\
\hline IV & $176(49,2)$ \\
\hline \multicolumn{2}{|l|}{ Tipo de primer tratamiento } \\
\hline Ninguno & $30(8,4)$ \\
\hline Cirugía & $13(3,7)$ \\
\hline Quimioterapia & $187(52,8)$ \\
\hline Radioterapia & $8(2,2)$ \\
\hline Paliativo & $116(32,4)$ \\
\hline \multicolumn{2}{|l|}{ Supervivencia } \\
\hline Primer año & $86(24,8 \%)$ \\
\hline Segundo año & $33(9,5 \%)$ \\
\hline Tercer año & $14(4,0 \%)$ \\
\hline
\end{tabular}

PAAF: punción-aspiración con aguja fina.

Técnica pleural incluye tóracocentesis y/o biopsia pleural. 


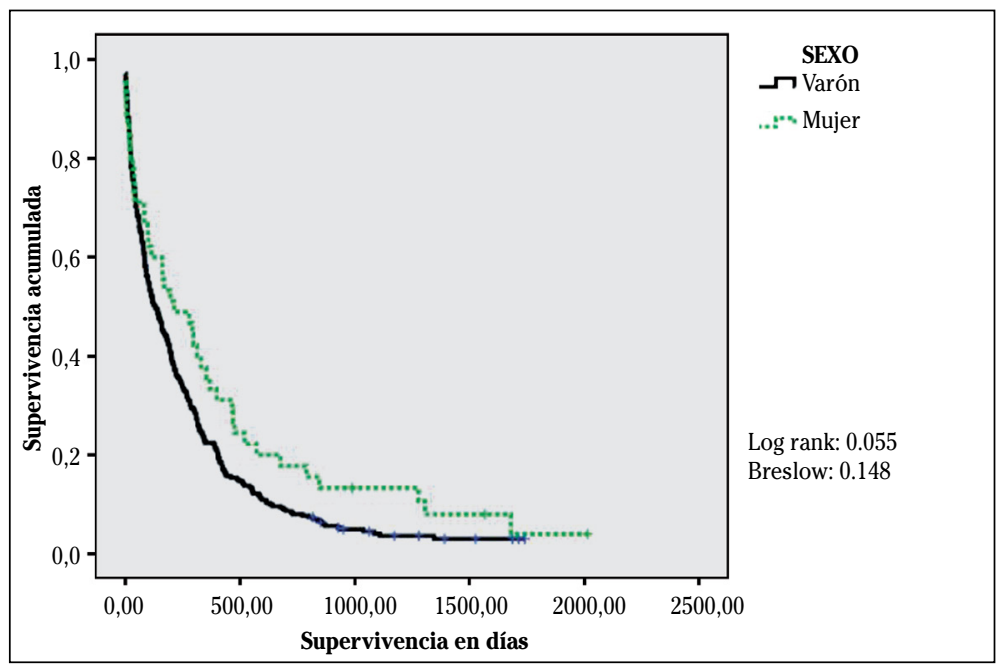

Figura 3. Supervivencia en función del sexo.

La supervivencia al primer año fue del $25 \%$, que se redujo al $4 \%$ al tercer año. En 11 casos no se pudo confirmar el estado vital, la mayoría de ellos por traslado fuera de la comunidad autónoma. En función del tipo histológico, la mayor supervivencia correspondió al epidermoide, con 351 días de supervivencia media, y la menor al microcítico con 220 días, aunque las diferencias no fueron significativas.
Por edades, por debajo de 70 años, tuvieron una supervivencia media de 316 días, significativamente superior a los 216 días en los de edad superior a la mediana por sexos, las mujeres presentaron una supervivencia media de 396 días, superior a los 247 días de los varones, aunque la diferencia no fue significativa (Tabla 4 ).

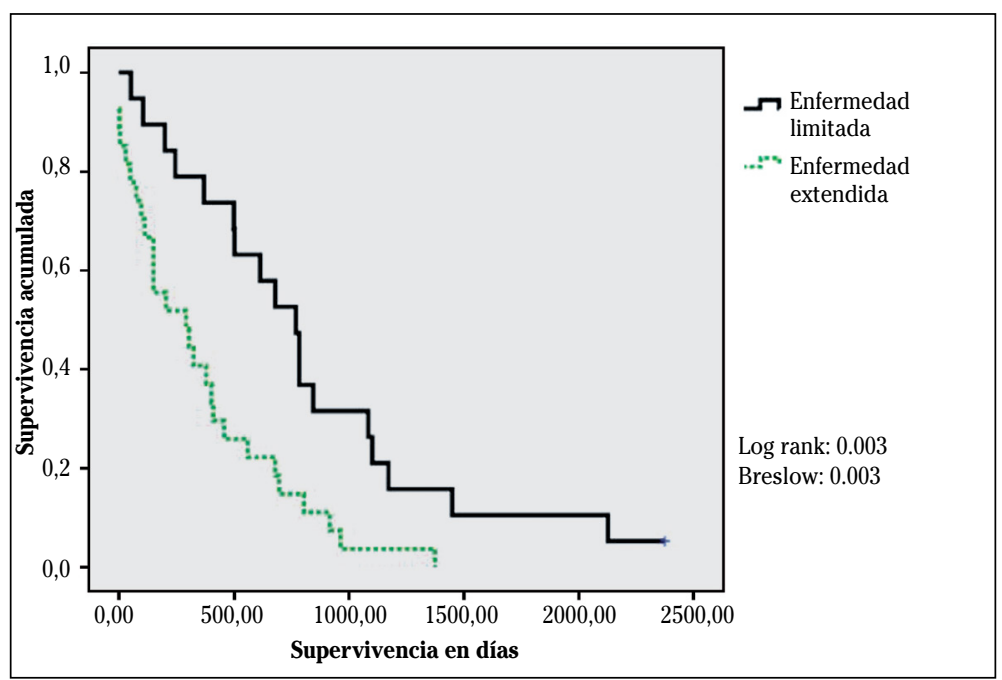

Figura 4. Supervivencia en función de estadíos. Cáncer de pulmón (células pequeñas). 
Tabla 4. Análisis de la supervivencia en función de edad, sexo, histología y estadio tumoral

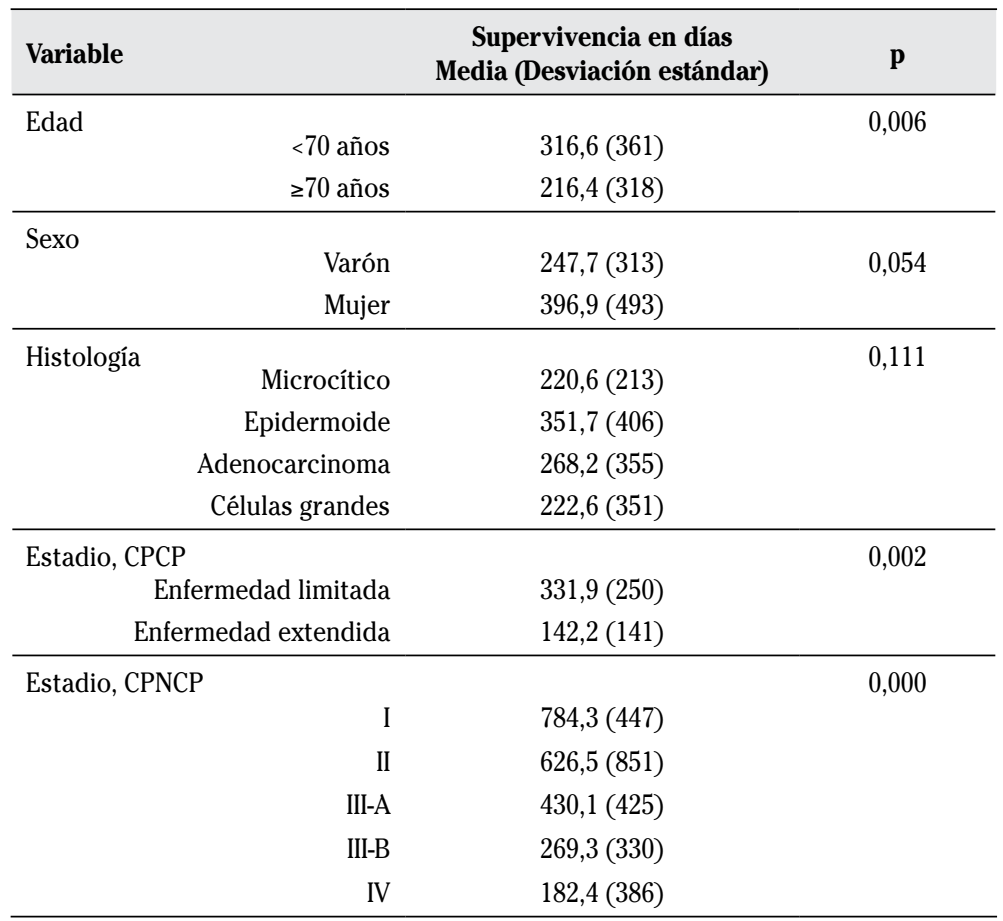

Supervivencia analizada con el método de Kaplan-Meier

CPCP: Cáncer de pulmón de células pequeñas

CPNCP: Cáncer de pulmón no de células pequeñas

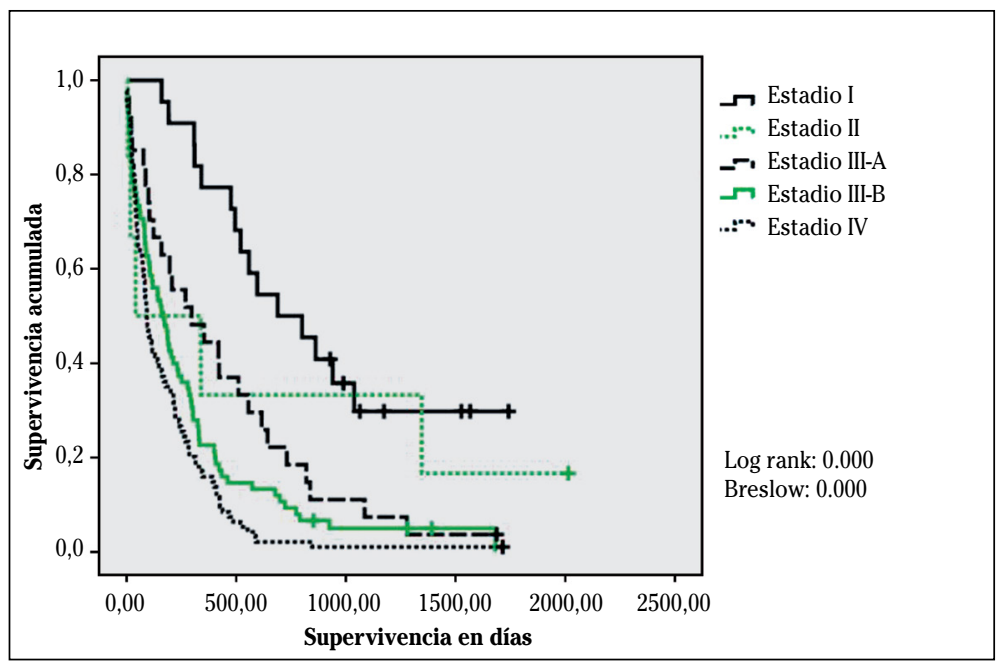

Figura 5. Supervivencia en función de estadíos. Cáncer de pulmón (no células pequeñas). 
En el CP microcítico se analizó la supervivencia en función de la presentación como enfermedad limitada o enfermedad extendida. Los pacientes con enfermedad limitada mostraron una supervivencia media de 332 días, significativamente mayor que los 142 días de los pacientes con enfermedad extendida (Tabla 4).

En función del estadio, analizamos la supervivencia del CP no microcítico estableciendo 5 grupos, estadio I, estadio II, estadio III-A, estadio III-B y estadio IV. La supervivencia se redujo significativamente con la progresión en el estadiaje, desde los 784 días en el estadio I, hasta los 182 días del estadio IV (Tabla 4).

\section{DISCUSIÓN}

La incidencia de $\mathrm{CP}$ en nuestra población muestra una situación claramente diferente en cada sexo. En los varones nos situaría entre el tercil superior de incidencia comparado con la población mundial; aunque, en el sexo femenino, estaríamos situados en el tercil inferior de inciden$\mathrm{cia}^{18}$. Esto parece un reflejo, al menos en parte, de la tardía incorporación de las mujeres de España al tabaquismo ${ }^{19}$.

Sin embargo, parece estar produciéndose un cambio en la distribución por sexos del CP en nuestra comunidad, ya que en este trabajo el $12,6 \%$ de los pacientes con CP fueron mujeres. En publicaciones previas incluyendo poblaciones cercanas a la nuestra, la proporción de mujeres fue muy inferior. En un trabajo que incluía pacientes diagnosticados de CP en los años 1995 y 1996, las mujeres suponían el 4,5\% de los $\operatorname{casos}^{5}$. En otra publicación, incluyendo casos diagnosticados entre 1997 y 1999, la población femenina era el 7,3\% del total de pacientes ${ }^{4}$. Esta tendencia es similar a la observada en otras comunidades españolas, con reducción progresiva de la ratio varones/mujeres ${ }^{2}$.

La relación del tabaco con el CP está bien establecida, pero en los últimos tiempos se está prestando cada vez mayor atención al CP en no fumadores ${ }^{20}$. La OMS estima la incidencia de CP en no fumado- res en un $25 \%$ del total de casos, aunque con amplias diferencias entre países y se$\operatorname{xos}^{20}$. En nuestro trabajo casi el $18 \%$ de los casos de $\mathrm{CP}$ se presentaron en pacientes nunca fumadores, muy superior al $6,1 \%$ referido por García-Prim y col y al 5,3\% de Montero y $\mathrm{col}^{4,5}$. En un estudio multicéntrico español, con datos recogidos en el año 2003 , la tasa de nunca fumadores fue del $9,6 \%$, también claramente inferior a la de nuestros pacientes ${ }^{6}$.

La exploración más frecuente para la obtención del diagnóstico fue la fibrobroncoscopia, que nos permitió el diagnóstico del 41,6\% de los casos. Este procedimiento suele ser el más frecuente en todas las series de estudio diagnóstico de $\mathrm{CP}^{5,7,8}$.

El tipo histológico más frecuente fue el epidermoide, en el $35,2 \%$ de los casos, seguido del adenocarcinoma en el 23,7\% de pacientes. Este predominio de la histología epidermoide se presenta en todos los estudios recientes realizados en nuestro país $^{4-10}$. En el sexo femenino la distribución de los tipos histológicos es diferente, siendo $59 \%$ de los casos adenocarcinomas, hallazgo coincidente con otras poblaciones $^{4,7,10,21}$. En USA el adenocarcinoma ya es el tipo histológico más frecuente en ambos $\operatorname{sexos}^{12}$, y en Europa también se está incrementando su incidencia relativa ${ }^{11}$.

Se plantean diversas causas para justificar el cambio en la distribución relativa de los tipos histológicos. Por una parte, estaría el aumento del número de casos en no fumadores, ya que el tabaco está más relacionado con el epidermoide y el microcítico $^{22}$. Otra posible explicación es la mayor incidencia en mujeres, en las cuales el adenocarcinoma es más prevalente ${ }^{10}$.

Los diagnósticos se realizan en estadios avanzados, estando en más del 78\% de los pacientes en estadio III-B o IV. Esta proporción de pacientes en estadios avanzados es claramente superior a la de otros estudios españoles, en los cuales el porcentaje de pacientes en estadios III-B y IV oscila entre el 49 y el $69,5 \%$, 7 .9.

También es avanzada la edad al diagnóstico con una media de 69 años, superior a publicaciones españolas recientes, donde oscila entre 65 y 68 años ${ }^{4,5,7,9}$. 
El tratamiento más frecuente es la quimioterapia, que se utilizó como primer tratamiento en el 53\% de pacientes; siendo la cirugía el tratamiento inicial en el 3,7\% de casos. Esta tasa de tratamiento quirúrgico es inferior a la media de las series publicadas $^{4,5,7-9,23-26}$; aunque en otras poblaciones son incluso inferiores. En un estudio multicéntrico español desarrollado en el año 2003 la tasa de resección en uno de los hospitales incluidos era del $2,5 \%{ }^{6}$. Asimismo, en un estudio inglés desarrollado entre los años 2004-2006 la tasa de tratamientos quirúrgicos era del $3 \%$ en alguno de los Primary Care Trust incluidos ${ }^{27}$. En este caso la tasa de resecciones podría estar sobreestimada en comparación con las de nuestra población, dado que solo incluyen pacientes con NSCLC ${ }^{27}$.

Lo avanzado de la edad y del estadio al diagnóstico justifica, al menos en parte, el bajo porcentaje de pacientes tratados quirúrgicamente. Es conocida la amplia variabilidad en el tipo de tratamiento entre distintas áreas geográficas. Así, en un estudio prospectivo multicéntrico español, incluyendo todas las cirugías como primer o segundo tratamiento, la tasa de tratamientos quirúrgicos oscila entre el 2,5 y el $20,6 \%{ }^{6}$. En otro trabajo comparando, con la misma metodología, dos áreas sanitarias de países diferentes, el porcentaje de pacientes tratados con cirugía va del $7 \%$ en Teesside-Inglaterra al $24 \%$ en Varese-Italia $^{28}$.

Diversos factores pueden justificar esta variabilidad. Por una parte, factores dependientes del paciente: estadio tumoral, edad, comorbilidad o aceptación de la cirugía; por otra parte, factores dependientes del cirujano, con mayor o menor disponibilidad a intervenir pacientes con pronósticos menos favorables (pobre función pulmonar o edad avanzada). En tercer lugar pueden influir factores institucionales como la disponibilidad de servicio de Cirugía Torácica ${ }^{23,26,27,29}$. En nuestro caso pudo influir el hecho de no disponer de servicio de Cirugía Torácica en nuestro hospital, debiendo derivarse los pacientes a otros hospitales.
La supervivencia al primer año fue del $25 \%$, y se redujo al $4 \%$ al tercer año del diagnóstico. La supervivencia de nuestra población es de las más bajas entre las series publicadas de países occidentales. En el primer año encontramos algunas publicaciones con tasas de supervivencia incluso menores, como el $23 \%$ registrado en un área de Inglaterra y el $21 \%$ en otro estudio escocés ${ }^{28,30}$; aunque en la mayor parte de estudios se refieren supervivencias en el primer año superiores, entre el $29 \%$ y el $48 \% \%^{4,10,11,25}$. El hecho de que nuestros pacientes sean de mayor edad, se diagnostiquen en estadios de la enfermedad más avanzado, y presenten menores tasas de tratamientos quirúrgicos, podrían ser algunos de los factores condicionantes de estas bajas tasas de supervivencia.

Como limitación de nuestro estudio, cabe indicar que no hemos podido incluir los casos diagnosticados y tratados en la medicina privada, por no tener contacto asistencial con el hospital. Dadas las características de la asistencia sanitaria en nuestro país, universal y gratuita, y la complejidad del proceso diagnóstico-terapéutico del $\mathrm{CP}$, es poco probable que el número de casos potencialmente perdidos en la asistencia privada supongan un cambio relevante en los resultados del estudio.

En conclusión, el diagnóstico del $\mathrm{CP}$ en nuestra población se lleva a cabo en fases avanzadas de la enfermedad, la tasa de resecciones quirúrgicas es baja y la supervivencia es pobre. La enfermedad es más frecuente en los varones que en las mujeres, especialmente si son fumadores, aunque la incidencia en el sexo femenino y en nunca fumadores es superior a otras poblaciones de nuestro entorno. Asimismo, a la vista de estos resultados, parece necesario profundizar en el análisis de las causas relacionadas la elevada edad y el avanzado estadio de la enfermedad tumoral en el momento del diagnóstico, que nos permitan optimizar esta situación. La mejoría en estos factores podría incrementar la tasa de tratamientos quirúrgicos y con ello prolongar la supervivencia de estos pacientes. 


\section{BIBLIOGRAFÍA}

1. Cabanes A, Vidal E, Aragonés N, Pérez-Gómez B, Pollán M, Lope V et al. Cancer mortality trends in Spain: 1980-2007. Ann Oncol 2010; 21 Suppl. 3: iii14-20.

2. Sánchez MJ, Payer T, De Angelis R, Larrañaga N, Capocaccia R, Martinez C. CIBERESP Working Group. Cancer incidence and mortality in Spain: estimates and projections for the period 1981-2012. Ann Oncol 2010; 21 Suppl 3 : iii30-36.

3. Chirlaque MD, Salmerón D, Ardanaz E, Galceran J, Martínez R, Marcos-Gragera R et al. Cancer survival in Spain: estimate for nine major cancers. Ann Oncol 2010; 21 Suppl 3: iii21-29.

4. Prim JM, Barcala FJ, Esquete JP, Reino AP, LóPEZ AF, CuAdRADo LV. Lung cancer in a health area of Spain: incidence, characteristics and survival. Eur J Cancer Care (Engl) 2010; 19: 227-233.

5. Montero C, Rosales M, Otero I, Blanco M, Rodríguez G, Peterga $S$ et al. Cáncer de pulmón en el Área Sanitaria de A Coruña: incidencia, abordaje clínico y supervivencia. Arch Bronconeumol 2003; 39: 209-216.

6. de Cos JS, Miravet L, Abal J, Núñez A, Muñoz FJ, GARCíA $L$ et al. Lung cancer survival in Spain and prognostic factors: a prospective, multiregional study. Lung Cancer 2008; 59: 246254.

7. Jiménez Massa AE, Alonso Sardón M, Gómez GóMEZ FP. Lung cancer: how does it appear in our hospital? Rev Clin Esp 2009; 209: 110-117.

8. Hueto Pérez De Heredia J, Cebollero Rivas P, Cascante Rodrigo Ja, Andrade Vela I, Pascal MarTínEz I et al. Evaluation of the use of a rapid diagnostic consultation of lung cancer. Delay Time of Diagnosis and Therapy. Arch Bronconeumol 2012; 48: 267-273.

9. Gullón JA, Suárez I, Medina A, Martín A, Cabrera C, GonZÁlez IJ. Lung cancer: changes in epidemiology and survival. Rev Clin Esp 2012; 212: 18-23.

10. Salmerón D, Chirlaque MD, Isabel Izarzugaza M, Sánchez MJ, Marcos-Gragera R, Ardanaz E et al. Lung cancer prognosis in Spain: The role of histology, age and sex. Respir Med 2012; 106: 1301-1108.

11. Sagerup CM, Småstuen M, Johannesen TB, HeLLAND $\AA$, BRUSTUGun OT. Sex-specific trends in lung cancer incidence and survival: a population study of 40,118 cases. Thorax $2011 ; 66$ : 301-307.
12. Kligerman S, White C. Epidemiology of lung cancer in women: risk factors, survival, and SCREENING. AJR Am J Roentgenol 2011; 196: 287-295.

13. World Health Organization.The World Health Organization histologycal typing of lung tumors. Second edition. Am J Clin Pathol 1982; 77: 123-136.

14. MountaIN CF. Revisions in the internacional system for staging lung cancer. Chest 1997; 111: 1710 .

15. Rami Porta R, Duque Medina JL, Hernández Hernández JR, López Encuentra A, Sánchez de Cos Escuin J. Normativa actualizada (1998) sobre el diagnóstico y estadificación del carcinoma broncogénico. Recomendaciones SEPAR. Arch Bronconeumol 1998; 34: 437-452.

16. Charlson ME, Pompei P, Ales KL, MacKenzie CR. A new method of CLASSIFYING prognostic comorbidity in longitudinal studies: development and validation. J Chronic Dis 1987; 40: 373-383.

17. Oken MM, Creech RH, Tormey DC, Horton J, Davis TE, McFadden ET et al. Toxicity and response criteria of the Eastern Cooperative Oncology Group. Am J Clin Oncol 1982; 5: 649-655.

18. Jemal A, Bray F, Center mM, Ferlay J, Ward E, FormAn D. Global cancer statistics. CA Cancer J Clin 2011; 61: 69-90.

19. Shafey O, Fernández E, Thun M, Schiaffino A, DoLWICK $S$, COKKINIDES V. Cigarette advertising and female smoking prevalence in Spain, 19821997: case studies in International Tobacco Surveillance. Cancer 2004; 100: 1744-1749.

20. Couraud S, Zalcman G, Milleron B, Morin F, SouQUET PJ. Lung cancer in never smokers-a review. Eur J Cancer 2012; 48: 1299-1311.

21. Wheatley-Price P, Blackhall F, Lee SM, Ma C, Ashcroft L, JitLaL M et al. The influence of sex and histology on outcomes in non-small-cell lung cancer: a pooled analysis of five randomized trials. Ann Oncol 2010; 21: 2023-2028.

22. Park SK, Cho LY, Yang JJ, Park B, Chang SH, Lee $\mathrm{KS}$ et al. Scientific Committee, Korean Academy of Tuberculosis and Respiratory Diseases. Lung cancer risk and cigarette smoking, lung tuberculosis according to histologic type and gender in a population based casecontrol study. Lung Cancer 2010; 68: 20-26.

23. Bendzsak A, Nenshi R, Darling G, Schultz SE, GunRaj N, Witton AS et al. Overview of lung cancer surgery in ontario. Ann Thorac Surg 2011; 91: 361-366. 
24. Beattie G, Bannon F, McGuigan J. Lung cancer resection rates have increased significantly in females during a 15-year period. Eur J Cardiothorac Surg 2010; 38: 484-490.

25. Skaug K, Eide GE, GulsviK A. Predictors of longterm survival of lung cancer patients in a Norwegian community. Clin Respir J 2011; 5: 50-58.

26. Svensson G, Ewers SB, Ohlsson O, Olsson H. Prognostic factors in lung cancer in a defined geographical area over two decades with a special emphasis on gender. Clin Respir J. 2012, en prensa.

27. Riaz SP, LÜChtenborg M, Jack RH, Coupland VH, LinKLATER KM, PeAKe MD et al. Variation in surgical resection for lung cancer in relation to survival: population-based study in England 2004-2006. Eur J Cancer 2012; 48: 54-60.
28. Imperatori A, Harrison RN, Leitch DN, Rovera F, Lepore G, Dionigi G, et al. Lung cancer in Teesside (UK) and Varese (Italy): a comparison of management and survival. Thorax 2006; 61: 232-239.

29. Berglund A, Lambe M, Lüchtenborg M, Linklater K, Peake MD, Holmberg L, et al. Social differences in lung cancer management and survival in South East England: a cohort study. BMJ Open 2012; 2(3).

30. Gregor A, Thomson CS, Brewster DH, Stroner PL, Davidson J, Fergusson RJ et al; Scottish Cancer Trials Lung Group; Scottish Cancer Therapy Network. Management and survival of patients with lung cancer in Scotland diagnosed in 1995: results of a national population based study. Thorax 2001; 56: 212-217. 